\title{
Pavlovian conditioning of sexual arousal: Unsuccessful attempts with an ejaculatory US
}

\author{
EDWARD ZAMBLE, G. MARILYN HADAD, and JOHN B. MITCHELL \\ Queen's University, Kingston, Ontario, Canada
}

\begin{abstract}
Although it is commonly assumed that ejaculation is the effective reinforcer for sexual behavior, there is little evidence for its role in the establishment of any Pavlovian conditioned arousal responses. This report includes three experiments (with rats) that used ejaculation as the US. In the first, a paired conditioning group was compared with a random control; in the second, conditions were varied in testing to look for conditioning to background cues; in the third, extinction trials were given in an attempt to provide a more sensitive test for any conditioned response. There was no evidence for conditioning in any of these cases, leading us to question the role of ejaculation.
\end{abstract}

The belief that learning plays a major role in the control of sexual arousal seems to be almost universal in psychology. For example, Bermant and Davidson (1974, p. 4) said that "it is clear that the sexual behavior of some species, in particular nonhuman primates and man, is modifiable through the operation of environmental contingencies."'

Still, the evidence is remarkably weak for an assumption of such strength and commonality. A few studies have shown some limited effects of Pavlovian contingencies in copulatory situations (e.g., Farris, 1967). However, reliable evidence for any sizable CR based on sexual arousal in mammalian species is not readily available.

Most commonly it is assumed that conditioning develops through the pairing of situational cues with ejaculation. Although this has some face validity, given that ejaculation is seen as the endpoint of sexual responding, it remains to be demonstrated. The present experiments were performed in an attempt to provide some of the necessary evidence.

\section{EXPERIMENT 1}

If the function of sexual arousal is to energize the behaviors in the copulatory response chain, then the best way to measure the strength of a CS for arousal ought to be through observing its effects on copulation. Thus, we employed such direct measures.

In the first study, the design was made as simple as possible in order to demonstrate a conditioned response. One group of rats was given a signal predicting the opportunity to copulate to ejaculation; an unpaired control group was maintained for comparison. If sexual arousal can be

This research was supported by grants from the National Science and Engineering Research Council of Canada. Marilyn Hadad is now at the University of Toronto, and John B. Mitchell is now at Concordia University. Requests for reprints should be sent to Edward Zamble, Department of Psychology, Queen's University, Kingston, Ontario, Canada K7L 3N6. conditioned in this manner, two such groups should show an increasing divergence over trials.

\section{Method}

Subjects. This experiment used 20 male Long-Evans hooded rats, approximately 100 days old, with no sexual experience prior to the experimental situation. Also used were 60 ovariectomized females of the same strain. All animals were housed in individual cages in a room with reversed light-dark cycles, and had free access to food and water.

Apparatus. Mating trials were conducted in arena boxes $60 \times 60$ $\times 50 \mathrm{~cm}$ high. Three sides and the bottom were of plywood painted flat black; the fronts were of removable Plexiglas, and the tops were open. The arenas were located in a dimly lit small room.

Procedure. The male rats were divided randomly into experimental (Group E) and control (Group C) groups, each with 10 animals. Subsequently, two males were dropped from Group $\mathrm{C}$ because of failure to complete copulation on the first 3 days.

Behavioral estrus was induced in the females by injections of estrogen and progesterone (Adler \& Bermant, 1966) on a 5-day cycle. Each female was paired with two consecutive males, chosen randomly except that each male had an equal number of first and second accesses and no more than three in a row of one kind.

In Group E, the male was taken in his home cage to the mating room and placed in an arena alone for $10 \mathrm{~min}$ (CS), after which a receptive female was placed into the arena; they were then allowed to copulate until the male had ejaculated. In Group C, the CS waiting period was omitted, and the female was introduced into the arena immediately. Control males were also given 10-min periods in the mating arena in the absence of the female, at random times during the day (excluding the 40-min period preceding mating trials). Thus, a signal preceded the opportunity to copulate for Group E, but not for Group C.

Trials were given during the middle $6 \mathrm{~h}$ of the dark period in the animals' home room, that is, between 1200 and $1800 \mathrm{~h}$.

During copulation trials, each intromission was recorded. The session was ended soon after ejaculation: When the male had disengaged himself completely from the female and was engaged in postejaculatory grooming, he was returned to the home room. In all cases, the occurrence of ejaculation was verified by visual inspection for the presence of a semen plug. If the male had not achieved an intromission within 30 min of meeting the female or had not ejaculated within 45 min, the trial was ended. Noncopulation trials were eliminated from later analyses.

The original training procedure was followed for $\mathbf{2 0}$ daily trials, after which the two groups were reversed; that is, Group $C$ was given arena placement as a signal for the imminent arrival of the female, and Group $\mathrm{E}$ was given no signal. The reversal was carried out for 4 days.

\section{Results}

From the data recorded, three measures of copulation 
were taken for analyses. Intromission latency (IL) is the time from the introduction of the female to the first recorded intromission. Ejaculatory latency (EL) is the time from the first intromission to ejaculation. These two statistics are the primary measures, but the total number of intromissions (NI) was also calculated, although its interpretation must be somewhat tentative given the uncertainties of visually distinguishing true intromissions from mounts without intromission.

Figure 1 shows mean EL for both groups over the course of training. The performance of all animals improved over trials, reaching an asymptote after about six trials, and the curves for the two groups are roughly parallel. Group E was faster to reach ejaculation throughout the 20 training trials, and inspection of the raw data indicates this difference even on the first trial.

Data from preliminary work on the latency measures had been skewed with some heterogeneity of variance. Therefore, they were transformed logarithmically before analyses. An analysis of variance confirmed the lower EL scores for Group E apparent in Figure $1[\mathrm{~F}(1,16)=4.80$, $\mathrm{p}<.05$ ], and the visible improvement over trials was also reliable $[F(16,304)=7.04, p<.01]$. However, the interaction between trials and conditions did not approach significance $(p>.2)$. Thus, there were differences between groups, but these differences were not the result of training.

The pattern during training for IL (not shown) was very similar. The analysis of variance showed that males in Group E began copulation more quickly than control subjects $[F(1,16)=4.85, p<.05]$ and also that scores decreased over trials $[\mathrm{F}(16,304)=10.27, \mathrm{p}<.01]$. Again, the interaction did not approach significance.

Finally, the results for NI were very similar, with su-

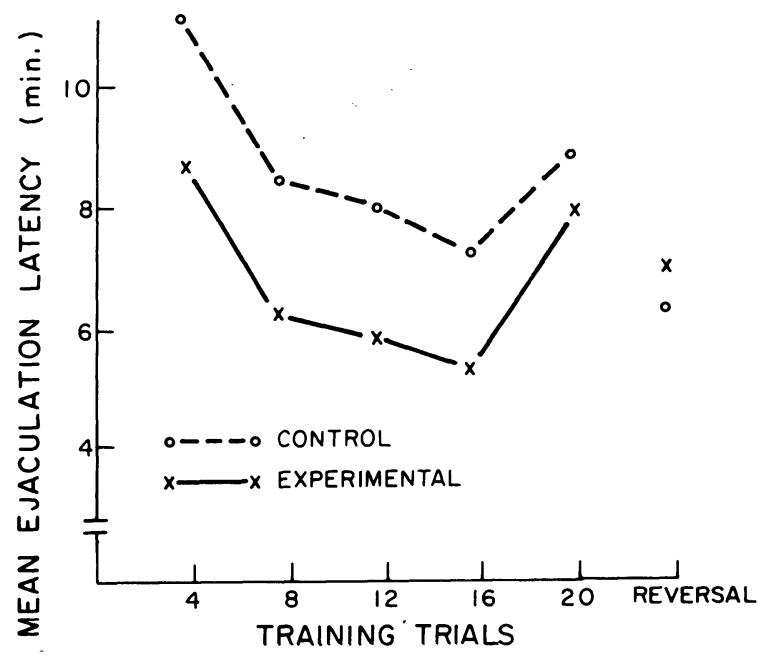

Figure 1. Mean ejaculation latencies across trials for the two groups in Experiment 1. In order to maintain comparability with the data for which statistical analyses are presented, the values shown are geometric means, that is, antilogs of the mean log values. perior performance for Group E. Both the difference between groups $[\mathrm{F}(1,16)=5.31, \mathrm{p}<.01]$ and the improvement over trials $[\mathrm{F}(19,304)=3.86, \mathrm{p}<.01]$ were significant, but the interaction did not approach reliability.

When conditions for the two groups were reversed, the superiority of Group E disappeared. On EL, the mean score for Group C improved, but there was no substantial change for Group E, as may be seen in Figure 1. As a result, an analysis of variance for all reversal days combined showed no significant differences.

On the IL measure after reversal, Group C improved and Group $\mathrm{E}$ became slower, and as a result the differences were statistically significant $[\mathrm{F}(1,16)=4.40, \mathrm{p}<$ .05]. Thus, for IL, the training difference was clearly reversed when signaling conditions were switched.

Analyses of the number of intromissions during reversal showed only some convergence between the groups, and no differences were significant.

\section{Discussion}

This experiment attempted to establish classical conditioning by pairing a signal with the opportunity to copulate to ejaculation. During the training stage, there were significant differences between the two groups on all measures. However, these differences cannot be attributed to conditioning, because they were apparent on the 1st day of training and changed immediately with reversal. Thus, the differences between groups indicate some sort of stimulus effect rather than conditioning.

However, if the differences between the groups do not indicate conditioning, there were also large changes in both groups over training trials. These may have been simply the result of the rats' learning to copulate (Dewsbury, 1969), or it may be that conditioning took place in both groups. All animals had to be taken from their home room, transported to a testing room, and placed in the mating arena. The associated set of background cues, or any part of it, may have served as a CS for both groups alike, and such background conditioning could have produced the changes over trials seen in both groups in this study. Thus, although the initial improvement over trials commonly is seen with copulation, one can hypothesize that it shows a Pavlovian effect. This hypothesis can be tested with an experimental design that is particularly sensitive to conditioning to general situational cues.

\section{EXPERIMENT 2}

Since it was hypothesized that conditioning could develop with background cues alone, we eliminated the overt signaling period in this experiment. In order to test the effects of background, the subjects were given copulation trials in one of two different environments.

In the test stage, each group was split, and the subjects were tested in either the original or the alternative location. If the sexual response were conditioned to background cues, one would expect that performance in the original location (with the effective CSs) would be better than that in the new location, in which conditioning could not have occurred. Thus, the differences between copulation in the original environment and copulation in the new location should measure the effects of conditioning.

\section{Method}

Subjects. Sixteen naive male rats, of the same strain as those in the previous experiment, were used. They were selected from an original pool of 23 , as described below. Female rats were used under the same 
conditions as in Experiment 1, and maintenance conditions were also the same.

Apparatus. The arenas used in this study were the same as those used previously. Males were housed in home cages twice the usual size, approximately $90 \times 20 \times 20 \mathrm{~cm}$. The home room was dimly illuminated in the dark part of the light cycle to allow the experimenter to see.

Procedure. The training stage used two groups: One was trained in the mating arena (Group A), and the other in the home cages (Group HC). Since pilot observations had indicated that males were more likely to copulate in their home cages, 14 rats were assigned randomly to Group A and only 9 males to Group HC. Subsequently, 6 were dropped from Group A for failure to copulate on the 1st training day, and only 1 was dropped from Group HC, leaving each group with 8 subjects.

In Group A, the subjects were first placed in the mating arena alone for a habituation period of $20 \mathrm{~min}$. On the following day, the male was transported to the mating room and immediately placed into the mating arena with a receptive female, and they were allowed to copulate. In Group HC, the female was placed directly into the male's home cage. The trial ended when ejaculation had occurred or when the maximum time had expired, as in Experiment 1.

This procedure was followed for 6 days, that is, until the males had reached an asymptotic level of responding. After this training stage, Group $\mathrm{HC}$ was given a 20 -min habituation period in the mating arena, and testing began.

For testing, each of the original groups was split: Half of the subjects were tested in the original training situation, and the other half in the alternative environment. Otherwise, test trials were identical to those in training. There were 3 test days.

\section{Results}

Training data were very similar to those from the first few trials of Experiment 1. As expected, both EL and IL showed improvement over trials [for EL, $\mathrm{F}(14,70)=$ $5.35, \mathrm{p}<.01$; for IL, $\mathrm{F}(14,70)=4.96, \mathrm{p}<.01]$.

The data of primary interest in this study were those in the test stage. Analyses were done on each measure for the 3 test days combined and for the 1st test day alone; subjects tested in their original training situation were compared with those switched to a new mating environment. There were no significant differences between groups on any measure. Thus, changing the environment produced no visible effect on copulatory performance.

\section{Discussion}

The subjects in this experiment showed the expected improvement in copulation during the training trials. If this were attributable to facilitation by a conditioned response to environmental cues, then changing the environment during the test stage should have led to disruption of responding. This did not occur. There was no indication that copulation in a new environment was in any way different from that in the original training situation.

\section{EXPERIMENT 3}

Despite the results of Experiment 2, it is still possible that conditioning was active in the first two experiments. If the effective CSs are irremovably embedded into the background environment, for example, the sight of the experimenter, then the manipulations in Experiment 2 would not have disturbed them. Thus, Experiment 2 might show only that the immediate environmental cues are not those active in the situation. If this is so, then any design that looks primarily at acquisition will have great difficulty, since the salient cues are practically impossible to control. Therefore, the next study looked at extinction of the hypothetical conditioned responses.

Regardless of the cues that control a response, its effect should be reversible with extinction operations. This experiment tested such a prediction. We also included a designated CS again to see whether it had any effects distinguishable from those of the background.

\section{Method}

Subjects. Thirty-five male rats, like those in the previous experiments, were used; they were chosen from an original pool of 60 , after a copulation pretest, as described below. Forty-eight female rats were also used, as before.

Apparatus. Mating arenas were the same as before. For CS presentations, we used white plastic $28 \times 18 \times 13 \mathrm{~cm}$ tubs with metal grid tops and bottoms covered with sawdust. These were chosen to present the subjects with a compound of stimuli in several modalities.

Procedure. Potential subjects were first pretested for the ability to copulate to ejaculation; those that did not begin copulation within $20 \mathrm{~min}$ and reach ejaculation within $30 \mathrm{~min}$ were discarded.

In the conditioning stage, all subjects were given the same treatment. The male was placed in the CS tub for $10 \mathrm{~min}$, after which he was carried to the mating room and placed into the arena with a female. They were left until the male had ejaculated, after which he was returned to the home room. Trials on which copulation to ejaculation did not occur within $30 \mathrm{~min}$ were considered null trials, and replacement trials were given.

There were 10 conditioning trials. In order to minimize null trials, the subjects were given a day of rest after every two trials. Data were recorded on the last conditioning trial, as baseline measures.

After conditioning, the subjects were divided into four groups by a stratified random process: The groups were given 5, 10, 20 or $30 \mathrm{ex}-$ tinction trials, respectively. We used 5 as the smallest number given, because preliminary work had convinced us that no changes from conditioning were visible at this point. There were eight subjects in the 5trial group and nine in each of the others.

An extinction trial consisted of a 10-min placement in the CS box, followed by a 15 -min placement in the mating arena without a female. The time of exposure was chosen to be slightly greater than the total time in the arena on a copulation trial. Thus, extinction trials were very similar to training trials, except for the absence of the female.

Following the completion of extinction trials, each group was given two copulation test trials. On the first, the CS was given before the male was placed into the arena with a female. The second test was a no-CS trial: The male was placed into the arena with the female without a prior signal. This was included to test for the effects of background stimuli alone. On both tests, copulation data were recorded.

\section{Results}

Visual inspection of the data did not indicate any sizable effects of extinction trials on any of the measures used. Although all subjects showed copulation times that were less than those in the pretest, there was no trend apparent across the number of extinction trials, and no group showed much change after extinction training from the levels of the last training trials.

Analyses of variance were performed, and no terms for any measure were even close to statistical significance.

\section{Discussion}

This experiment makes it difficult to argue that the copulation practice effect shows a conditioned response, unless one maintains that it 
is not subject to extinction-a most unlikely hypothesis. Therefore, we must conclude that we cannot see evidence for any conditioning process in our data.

\section{GENERAL DISCUSSION}

Taken together, the results of thèse experiments show that conditioning is difficult or impossible to demonstrate when the US consists of the entire mating sequence up to the point of ejaculation. It should be mentioned that we have performed several other studies in which we used different values for various conditioning parameters, for example, shorter CS-US intervals. In no case have we seen any good evidence of the existence of conditioned arousal based on pairings with ejaculation.

It is of course still possible that we have simply not found the correct parameters for this sort of conditioned response or that we have used ineffective stimuli as CSs. However, the variety of situations that do not show evidence for conditioning might be used to argue that ejaculation does not easily support a conditioned response. Our results are also in line with the curious lack of previous evidence in the literature. Certainly, the present paradigm does not seem a good candidate to account for anecdotal evidence on the power of acquired sexual arousal responses.

This conclusion should also be considered in the context of other studies in our laboratory that have shown powerful Pavlovian effects from a
CS for nonejaculatory sexual activity (Zamble, Hadad, Mitchell, \& Cutmore, in press). As stated there, it is more likely the theoretical expectations that ejaculation would work as an excitatory reinforcer that are defective, rather than any of the procedures used here.

\section{REFERENCES}

Adler, N. T., \& Bermant, G. (1966). Sexual behavior of male rats: Effects of reduced sensory feedback. Journal of Comparative and Physiological Psychology, 61, 240-243.

BERMANT, G., \& DAVIDSON, J. M. (1974). Biological bases of sexual behavior. New York: Harper \& Row.

DewsBury, D. A. (1969). Copulatory behavior of rats as a function of prior copulatory experience. Animal Behaviour, 17, 217-223.

FARRIS, H. E. (1967). Classical conditioning of courting behavior in the Japanese quail, Coturnix coturnix japonica. Journal of the Experimental Analysis of Behavior, 10, 213-217.

Zamble, E., Hadad, G. M., Mitchell, J. B., \& Cutmore, T. (in press). Pavlovian conditioning of sexual arousal: First- and secondorder effects. Journal of Experimental Psychology: Animal Behavior Processes.

(Manuscript received for publication November 16, 1984.) 